\title{
Towards a Virtual Coach for Boccia: Developing a Virtual Augmented Interaction Based on a Boccia Simulator
}

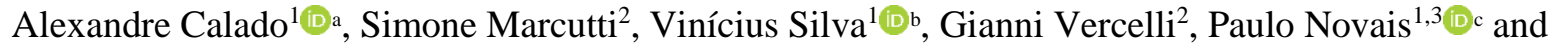 \\ Filomena Soares ${ }^{1}$ iDd \\ ${ }^{1}$ Algoritmi Centre, University of Minho, Guimarães, Portuga \\ ${ }^{2}$ Department of Informatics, Bioengineering, Robotics and Systems Engineering, University of Genova, Genoa, Italy \\ ${ }^{3}$ Department of Informatics, University of Minho, Braga, Portugal \\ alexandreluiscalado@gmail.com, simone.marcutti@edu.unige.it, a65312@alumni.uminho.pt, gianni.vercelli@unige.it, \\ pjon@di.uminho.pt, fsoares@dei.uminho.pt
}

Keywords: Boccia, Virtual Reality, Virtual Coach, Human Machine Interface

Abstract: Disability can be a factor that leads to social exclusion. Considering that involvement in society is paramount for a person with disability, participation in sports can be a powerful tool for inclusion. Based on this premise, the authors propose an intelligent virtual coach for Boccia to encourage the practice of this sport on persons with disabilities, while promoting social inclusion and shortening the learning curve for individuals new to the sport by learning about game strategy. The envisioned virtual coach will rely on Artificial Intelligence models, thus requiring the creation of large datasets, namely for ball placement and throwing movement recommendations. To answer these problems, this work is focused on the development of a Boccia simulator. With this simulator, it is possible to generate artificial gameplay images and allow the user to control an avatar with body tracking. Gesture recognition was implemented with a state-machine, thus enabling the player to throw the ball, with customizable physics, by performing one of two different throwing movements. This functionality can allow the recording of data describing the body movement associated with the placement of the ball in a certain position within the virtual court, which is essential for the proposed recommendation system.

\section{INTRODUCTION}

The World Health Organization estimates that, based on data from 2010, more than one billion individuals live with some form of disability worldwide. However, the term "disability" is one difficult to define due to its complexity. According to the International Classification of Functioning, Disability and Health (ICF) (World Health Organization, 2011), problems with human functioning can be organized in three areas: impairments, activity limitations and participation restrictions. Thus, disability can be interpreted as the difficulties encountered in any or all the three aforementioned areas, result of the interaction of health conditions with environmental and personal factors. Additionally, the United
Nations General Assembly (UNGA) has established that the condition of disability is a key factor of social exclusion (Fina, Cera, \& Palmisano, 2017).

The extensive work developed by John Pierson (Pierson, 2009) proposes the following broad definition for the term "social exclusion": "Social exclusion is a process that deprives individuals [...] of the resources required for participation in the social, economic and political activity of society as a whole. [...] Through this process people are cut off for a significant period in their lives from institutions and services, social networks and developmental opportunities that the great majority of a society enjoys.". Moreover, the United Nations (UN) defends that sutainable development is based on three dimensions: economic, social and economic (UN,

\footnotetext{
a https://orcid.org/0000-0002-2725-1067

b (D) https://orcid.org/0000-0000-0000-0000

c(D) https://orcid.org/0000-0002-3549-0754

d(D) https://orcid.org/0000-0002-4438-6713
} 
2013). Considering these remarks, one can understand the importance of the social aspect in the life of a person with disability. Thus, it is paramount to design and implement strategies to counteract the effect of disability on social exclusion and promote inclusion.

The use of sports can be a powerful tool for the inclusion of persons with disabilities, as it can promote social interaction amongst the participants. According to Burchel (Burchell, 2006), the participation in sports is important for socio-cultural integration and equity. Furthermore, besides the wellknown health-related benefitis, engaging in sports can contribute for the empowerment of persons with disabilites, as long as increasing their independence

The social importance of physical activity on the life of persons with disabilities is well stated in the Article 30 of the UN Convention on the Rights of Persons with Disabilities (CRPD), which addresses this subject by listing a set of measures to be taken by State Parties (United Nations, 2006). However, persons with disabilities often face barriers concerning participation in sports. According to Donnely \& Kidd (Donnelly \& Kidd, 2006), two of the most common causes for non-participation are the lack of early experience and the lack of awareness of how to include these individuals in sports.

Taking into account the aforementioned remarks, the authors propose a solution based on the game of Boccia to tackle social exclusion of persons with disabilities.

Boccia is a strategy-based precision ball game that has been developed for individuals with cerebral palsy. However, due to the dynamics of the game, it can be easily adapted to persons with other types of motor impairments or even mental ones. From 1984 that this game is considered a Paralympic Sport, which is an important fact to point out, since the Paralympic Games are one of the most relevant elite sporting events nowadays.

Although Boccia can be played individually, i.e. one against one, it can be played in teams of two or, more generally, of three players. Considering that it can be played in teams, Boccia is a game that promotes social interaction, as players of the same team need to communicate between each other to define a team strategy. Besides, it also promotes competitivity between teams, which have to stick together in order to reach a common goal and beat their opponents.

The authors propose to develop an artificial intelligence-based virtual coach for Boccia, already referenced in previous work (Calado, Marcutti, Vercelli, \& Novais, 2019). Although it is still a work in progress, the latter will be based on a virtual assistant, with whom the player can interact through voice commands, that will be capable of autosuggesting the best possible move for each player (i.e. the best position to place the Boccia ball), depending on the circumstances of the game. The virtual coach will also be able to suggest the most adequate way of performing the throwing movement, depending on the recommended position to place the ball within the court. These recommendations will be made by the virtual coach via visual and auditory feedback.

However, considering the possible use of deep learning approaches to build a reliable recommender system, a high amount of data is needed. Thus, it is necessary to build a large image dataset containing a wide variety of game situations in order to train a model with good generalization properties. Because Boccia is not as "mainstream" as other sports, such as football or tennis, it is difficult to acquire a large number of images from television broadcasts or from the internet, as there are no Boccia image datasets available, at least to the best of the authors' knowledge. This requires serious efforts to build a good dataset, an issue that was approached in previous work (Calado, Marcutti, et al., 2019). In the latter, a virtual Boccia simulator was developed with functionalities that enable the user to easily place each Boccia ball in any desired position within the virtual court and take a screenshot from any camera angle. This allows the convenient generation of images containing artificial Boccia game situations, thus substantially aiding in the construction of a consistent dataset. However, one must bear in mind that real game situations must also be present in the dataset.

The work described in this paper mainly focuses on new developments made for this simulator regarding the tracking of the player's body for controlling an avatar within the virtual court. To be more specific, a Microsoft Kinect v2 was used to capture the player's body data. The data stream is then used to make the virtual avatar replicate the movements performed by the player, allowing the latter to throw the Boccia balls inside the virtual court, in a similar fashion as he/she would in real-life.

This new functionality was added to the simulator with the purpose of understanding what may be the most appropriate throwing movement to place the ball in the desired position, inside the virtual court. With the propper parameters, the simulated throw can be a good approximation of a real one, thus, this tool can be used to record data for the training of models regarding the throwing movement recommendations made by the virtual coach. 
The present paper is divided in four sections. Section 2 addresses a literature review regarding existing virtual coaches and Boccia simulators. Section 3 describes the current virtual Boccia simulator system architecture, along with a preview of the virtual coach system architecture. Section 4 presents the preliminary results, showcasing the functionalities of the current implementation of the simulator. Finally, final remarks and future work are discussed in Section 5.

\section{LITERATURE REVIEW}

The concept of a virtual coach is no novelty, the idea of a personal device that can monitor, motivate and teach the user is very attractive for individuals who want to improve their fitness or their general health but do not want to hire a personal trainer, whether due to the cost or due to shyness. According to the review from Lowe et al. (Lowe \& ÓLaighin, 2012), some of the virtual coaching systems that are available in the market can be divided into three categories: smartphone applications, sensors devices and image processing devices. Smartphone applications use some of the sensors that are typically integrated in a smart phone (e.g.: GPS and inertial sensors) for monitoring physical activity, such as Google Fit and RunKeeper. Sensor devices can be considered systems that use a central controller, such as a smartphone, wristband or other embedded controllers, and external sensors. Nike+ and Polar systems are some of the existing solutions that fit in this category. Finally, image processing devices use cameras or similar solutions to monitor the movements and position of the user's body during physical activity, such as "Your Shape: Fitness Evolved", a game for Xbox 360 that uses Microsoft Kinect. In general, these virtual coaching systems can provide feedback to the user during or after the exercise, along with the possibility of creating a training program. Taking this into account, the system proposed in this work can be considered to fall into this category.

Besides the systems that can be found in the market, several other examples of virtual coaches regarding its application for physical activity motivation and monitoring can be found in the literature. For instance, Watson et al. (Watson, Bickmore, Cange, Kulshreshtha, \& Kvedar, 2012) developed an internet-based virtual computer agent with the goal of increasing the level of physical activity on overweight or obese individuals.
Ruttkay et al. (Ruttkay \& Van Welbergen, 2008) implemented an intelligent virtual agent that, besides presenting a sequence of exercises to be performed, it provides feedback based on utterances and animations to correct the exercise specific movements in real-time and also to motivate the user. Furthermore, it can also adjust its tempo to be in sync with the user. The results of a try-out showed that the users got engaged with this virtual coach, however, the feedback could be misleading or too general and the speech quality, along with the aspect of the avatar, should be improved. These are remarks to be taken into account when designing a similar system, in the sense that the interactions between the virtual coach and the user should resemble real interhuman interactions in order to motivate the user.

Additional approaches for correcting technical movements by a virtual agent were explored in other works, such as the one by Kelly et al. (Kelly, Healy, Moran, \& O’Connor, 2010), whom used a virtual coaching environment to improve the golf swing technique. In this case, the authors used forty-four reflective spherical markers (forty-one placed on anatomical landmarks and three on the golf club) to record the subject's golf swing movement in $3 \mathrm{D}$, by using an infra-red motion capture system. A 3D virtual avatar mimicking the subject's swing movement is then aligned and compared to the movements of a "coach" 3D virtual avatar, which are based on the average positions of multiple skilled golf players. Additionally, graphs can be used to analyse the joint angles throughout the swing movement and examine the differences between the "apprentice" and the "expert" player. Overall, both features can help the user to learn how to achieve the correct golf swing technique by adjusting his/her movement to a virtual coach.

Eyck et al. (Eyck et al., 2006) claims that the use of a virtual coach is capable of increasing the athletes' interest and enjoyment. Furthermore, it is a controlling factor in the sense that individuals increase their physical activity level to receive rewards and avoid negative consequences. However, further research is necessary to assess the long-term effect of this type of systems.

Regarding Boccia, as far as the authors' knowledge, no virtual coaching system was found in the literature. The same was observed for similar sports, such as pétanque, bocce or lawn bowls. However, several Boccia virtual simulators were found, which is closely related to the main focus of this work.

For instance, the Centro de Referencia Estatal de discapacidad y dependência in León, Spain, a social 
services centre focused on aiding persons with severe disability and dependence, has developed a Boccia simulator videogame to facilitate and promote sports practice (CRE Discapacidad y Dependencia, 2016). The game explains the Boccia rules and allows the user to throw the ball using a Nintendo Wii remote. Besides, it is possible to choose between three levels of ball hardness/softness, considers the different athlete categories and includes the option of using a ramp for launching the ball.

A company called Preloaded, in a collaboration with Channel 4, developed a 3D Boccia simulator for the general public in order to raise awareness for the Paralympic Sports (Edwards, 2012). It is a very complete videogame which is regularly used to teach schools about Boccia, and it features three modes: quick play, tournament and arcade. Quick play allows the user to play a game against another player on the same machine or against the computer AI. In tournament mode it is possible to play against the biggest names in Boccia, in a simulated tournament. Finally, arcade mode focuses on increasing skills, such as target practice.

Another example is the BocciaSim (Osório \& Sá, 2008), an open-source project started by two high school students. It focuses on a simple 2D Boccia simulator controlled with mouse and keyboard, which makes it less attractive to be played by users with disabilities due to its lack of intuitiveness and immersiveness.

That were also some works found in the literature regarding this subject, such as the work by Guedelha (Guedelha, 2007), which presents a basic version of the Boccia game controlled only by keyboard and mouse or joystick. Finally, Ribeiro (Machado Ribeiro, 2017) made efforts to create a realistic Boccia simulator focused on BC3 classification atheletes based on Unreal game engine, allowing accurate physics and realistic collision and ballistics.

As far as the authors' knowledge goes, no other Boccia simulator was found in the literature with the same purposes as the one presented in this paper, i.e. the creation of image and body data datasets.

It is noteworthy to mention that previous work by the authors also used Boccia as a context for promoting and monitoring physical activity on the elderly (Figueira et al., 2017; Silva et al., 2018). This included efforts regarding real-time game scoring (Calado, Silva, Soares, \& Novais, 2019; Leite, Calado, \& Soares, 2018), throwing movement detection (Calado, Leite, Soares, Novais, \& Arezes, 2018) and User-Interface design (Calado, Leite, Soares, Novais, \& Arezes, 2019).

\section{SYSTEM ARCHITECTURE}

This section describes the system architecture of the current implementation of the Boccia simulator. The predicted architecture for the virtual coach (which is a work in progress) is also presented in order to help the reader understand the role of the simulator in the overall system.

\subsection{A Preview of the Virtual Coach}

Figure 1 depicts a diagram regarding a preview of the architecture for the virtual coach system. The area-ofplay is captured, in real-time by a camera placed directly above the Boccia court. A deep learning model is then used to detect each of the Boccia balls within the camera Field-of-View (FOV) and sort them according to colour (red, blue or white), which was the target of previous work (Calado, Silva, et al., 2019). The distance between each red and blue ball and the jack (white ball) is then computed, based on their respective centroid coordinates, which allows the mapping of all balls within the area-of-play and the computation of the score for the current game situation. This data is then forwarded to each of both team's Strategy Learning Algorithm, a model that computes a recommendation regarding the optimal position to place the ball, providing team strategy aided by artificial intelligence.

For each suggested move there is an associated throwing movement that may facilitate the task of placing the ball in the desired position. A second algorithm can recommend a throwing movement that is more adequate for this task. The output from the latter can also allow the player, through a virtual avatar displayed on the Virtual Coach GUI, to see the representation of the suggested throwing movement, thus improving training.

However, the training of the two models, regarding ball positioning and throwing movement recommendations, requires a large amount of data. This is where the virtual Boccia simulator plays an important role, as its current features allow the easy generation of gameplay images and simulation of throws with realistic ball physics using real time body tracking data, which is also possible to be recorded.

The aforementioned recommendations are then conveyed to the virtual coach per se, an intelligent virtual agent capable of using the incoming data to give visual and audio feedback to the player, so the latter can make an informed decision about his/her next move or learn different types of Boccia strategies. 


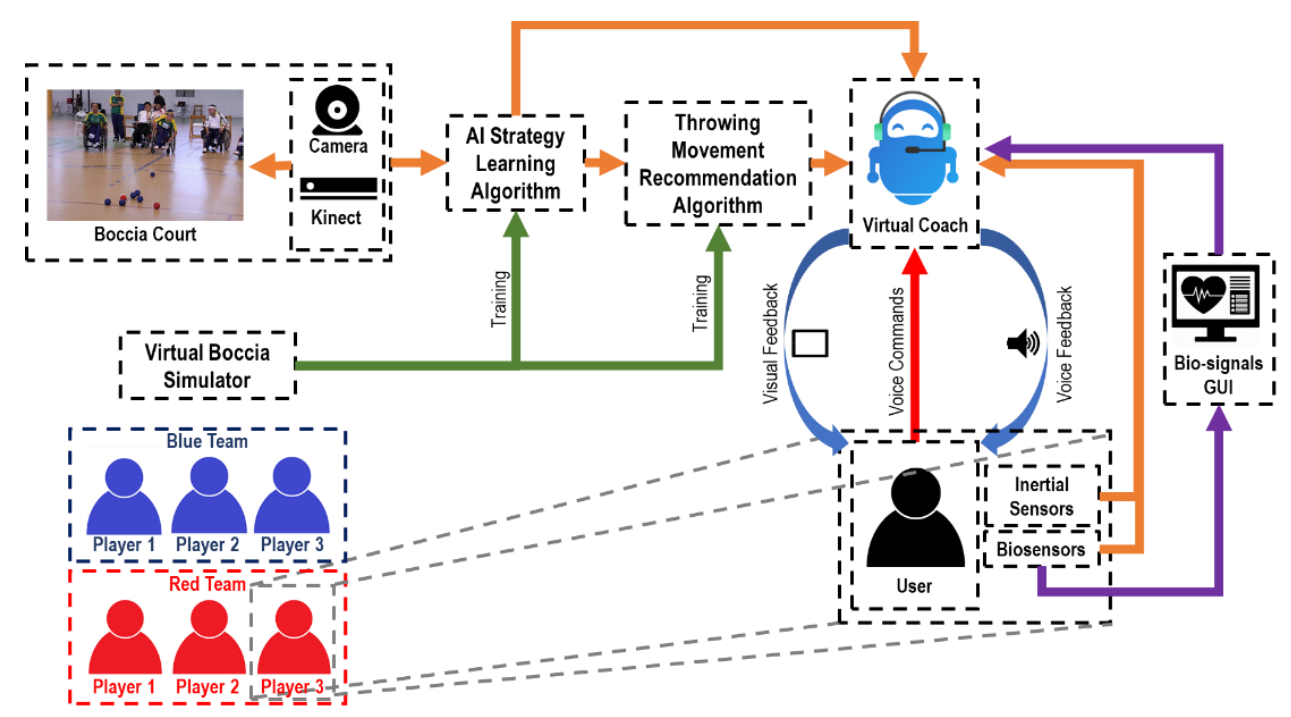

Figure 1: Preview of the virtual coach system architecture

The user will be able to dynamically interact with the virtual coach at any time by using voice commands and natural language processing. The virtual coach will be able to convey information to the player by using a display placed on the players' chair that will show the area-of-play covered by the camera, a virtual avatar, current game score and the suggested optimal ball position. Additionally, headphones can be used for audio feedback based on voice cues.

Moreover, different types of sensors will be used on the players in order to acquire various bio-signals, which the behaviour may trigger different virtual coach responses. Regarding the bio-signals GUI, the signals acquired by the biosensors, as well as the ones acquired by the inertial sensors, are stored in a data base and plotted for each game, or session, so the coach, or caregiver, can posteriorly analyse the player's physiological data and detect any possible anomalies.

\subsection{Virtual Boccia Simulator}

The current architecture of the Boccia simulator, the main focus of this work, can be observed in Figure 2. This system is composed by a Kinect sensor, a processing unit, and an external screen for displaying the graphic interface.

The Microsoft Kinect V2 (Microsoft, 2017) is used in order to track the user joints in real-time. This device is capable of tracking to up to 6 users and extract in real-time facial features, user joints position in the 3D space, and recognize gestures. The body tracking API outputs as skeleton model composed of 25 joints. In order to reliably work with Kinect in several platforms at the same time, a Node.js application that uses the Kinect2 library (Verweirder, 2015) was developed. This Node.js application, on the Windows OS machine, can receive the sensor data by using the official Kinect SDK and transmit the data to other platforms. The Kinect V2 is used to track the players and to recognize the two main gestures (underarm throw and upper arm throw) performed when throwing a ball during a Boccia match.

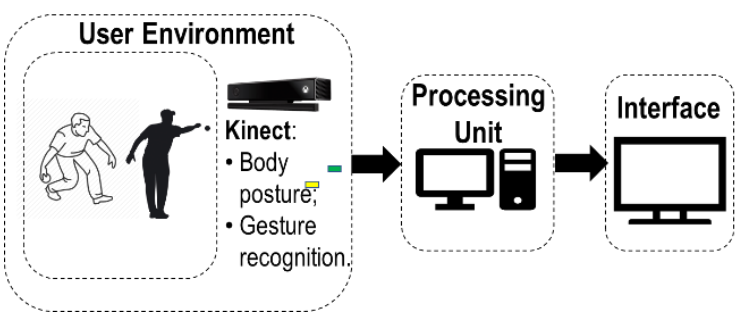

Figure 2: Proposed system architecture

\section{PRELIMINARY RESULTS}

The Boccia simulator was developed in Unity 3D game engine (Unity, 2019) and all of the dimensions used were set according to the real-world measures, including the court lines, ball dimensions and weight, and the avatar's height. In Figure 3, it is depicted a diagram with the main functionalities of the simulator. For better understanding of the Boccia game rules, the interested reader may refer to the official Boccia International Sports Federation documentation (BISFed, 2017). 


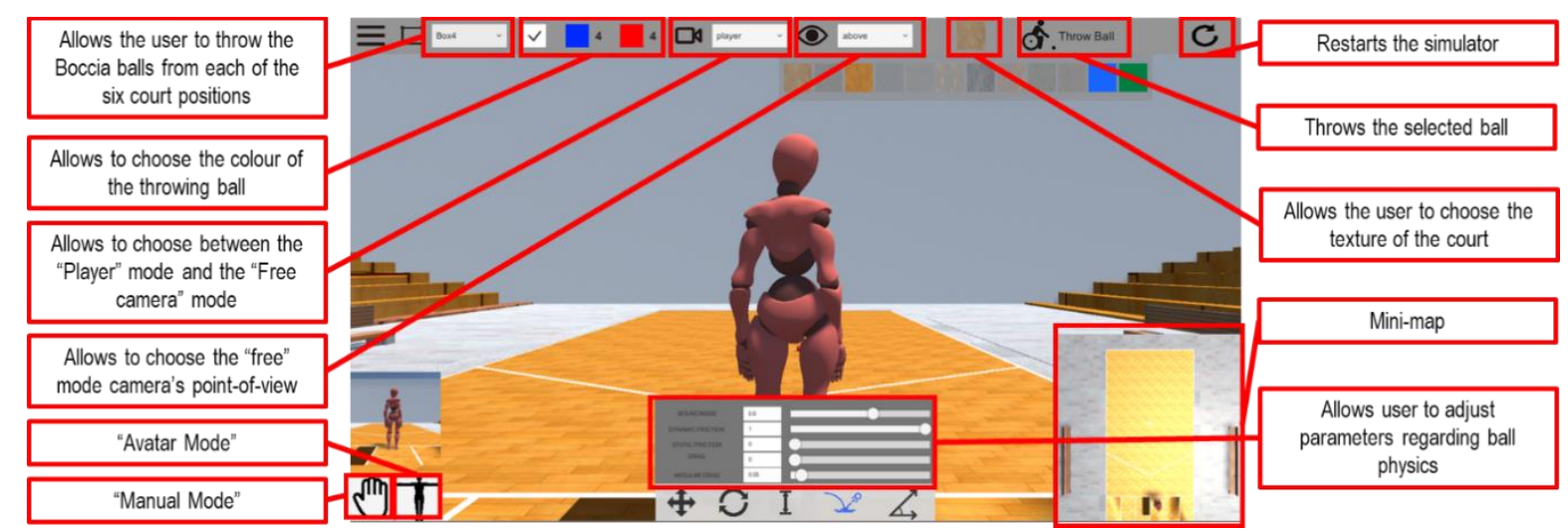

Figure 3: Overview of the most relevant features of the virtual Boccia simulator

A "free-camera" mode was implemented in previous work (Calado, Marcutti, et al., 2019), which allows the placement of Boccia balls anywhere inside the court and the taking of screenshots. The court is also customizable by changing the texture of the floor background by choosing one of twelve available textures as shown in Figure 3.

These customizations facilitate the extraction of images, augmenting the data to be used for the training of Artificial Intelligence algorithms referenced in section 3.1. An example of a game situation image generated by this feature can be observed in Figure 4.

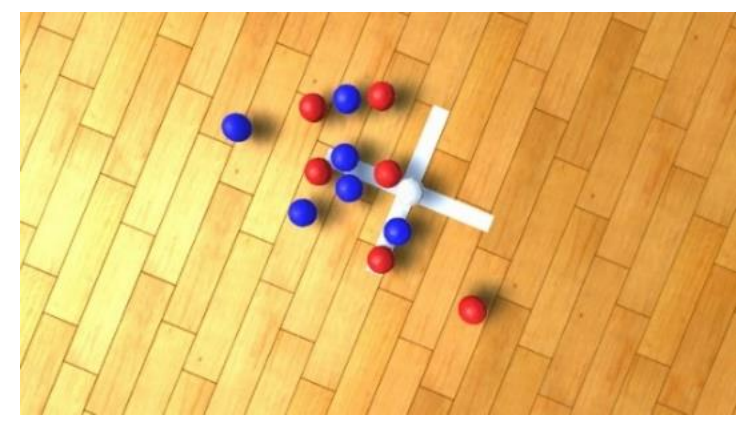

Figure 4: Example of an artificial game situation generated with the "free-camera" functionality

The new functionalities added to the simulator allow the user to select an "avatar mode", which enables the visualization of a virtual avatar mimicking the player movements in real-time. The used avatar was adapted from (Mixamo, 2019), Figure 5.

Additionally, a gesture recognition algorithm based on a state-machine was implemented (Figure 6 ), which detects when the player performed, with the right arm, one of the two most common throwing gestures in Boccia: underarm and overarm. A statemachine is a simple model to track the events triggered by external inputs. This is done by assigning intermediate states to decide what happens when a specific input comes, and which event is triggered.

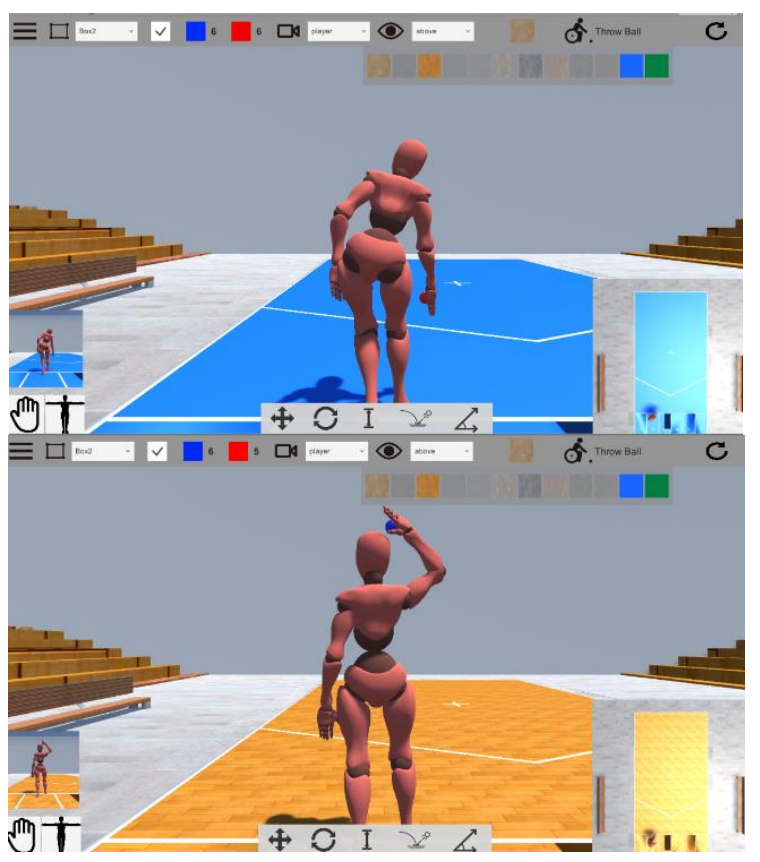

Figure 5: Up: Player performing the underarm throw; Down: Player performing the overarm throw

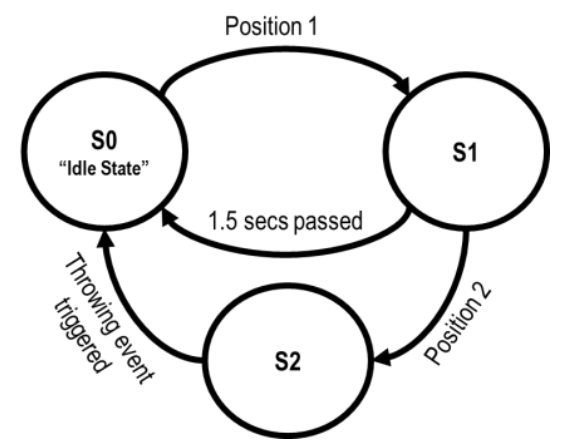

Figure 6: State-machine used for detecting throwing gestures from the Kinect body tracking data 
The implemented state-machine has three states. It is initialized on the idle state and passes to state S1 when the right arm is in a typical position for building momentum (Position 1 in the diagram), whereas, if the player's right arm is in a position that is typically assumed at the end of a throwing movement (Position 2 in the diagram), the state-machine passes to state $\mathrm{S} 2$. However, if Position 2 is not assumed before 1.5 seconds have passed after state $\mathrm{S} 1$ is activated, the state-machine returns to idle state ( $\mathrm{S} 0$ ).

As the state-machine reaches $\mathrm{S} 2$, a throwing event is triggered, and the ball is thrown according to the direction of the velocity vector of the avatar's hand joint at the time instant when state $\mathrm{S} 2$ is activated. The force at which the ball is thrown is calculated as being the mass of the ball (which is set to $275 \mathrm{~g}$ in the Unity world, in accordance with the game regulations) times the avatar's hand joint acceleration, allowing to simulate the trajectory on the virtual simulator using physics approximated to reality.

The parameters used for complying with the conditions set by Position 1 and 2 were based on the difference between the right hand and shoulder coordinates acquired by the Kinect.

Furthermore, as depicted in Figure 3, the ball physics are customizable by the user, which includes the following parameters used for adjusting the physics of a rigid body in the Unity's virtual world: bounciness, static friction, dynamic friction, drag and angular drag. Thus, the user can regulate these parameters to realistically simulate the throwing of balls with different levels of softness/hardness, as it is done in the game. Of course, the setting of these parameters depends on the disability and the associated level of the player.

Alternative to the "avatar mode", the user can select the "manual mode", where all the parameters related with ball throwing can be manually set, such as height, force magnitude and direction. Additionally, it also provides the option to position the ball anywhere inside the previously selected box in the court before throwing it.

\section{FINAL REMARKS AND FUTURE WORK}

The World Health Organization estimates that approximately $15 \%$ of the worldwide population lives with some form of disability. Having a disability can lead to social exclusion, therefore it is paramount to ensure that every individual with a motor or cognitive disability can be able to participate in society, to the largest extent possible.

Following this idea, the present work suggests the development of a virtual Boccia coach in order to tackle these issues. This tool aims to contribute for the inclusion and empowerment of individuals with motor or mental impairments. Additionally, a Virtual Boccia Simulator was developed to easily allow the creation of different types of game situations for the training of new players as well as, the virtual coach's Artificial Intelligence algorithms.

This work focused on new developments made on the simulator, namely the "avatar mode", enabling the visualization of a virtual avatar mimicking the player movements in real-time. Moreover, a gesture recognition algorithm based on a state-machine was implemented, which is able to detect when the player performs one of the two most common throwing gestures in Boccia.

Future work includes a continuous improvement of the simulator, by tackling the regularity of the virtual court's ground to better mirror the real-world conditions. The Kinect data will be recorded to later be used to train the virtual coach recommendation system. Additionally, it is intended to add wearable devices to the framework since it can provide more data to the virtual coach. The system level autonomy should also be increased by adding interactive machine learning enabling it to learn on the fly. This will allow the input of knowledge from professionals into the system in order to better train the virtual coach, improving the virtual coach recommendations.

\section{ACKNOWLEDGEMENTS}

This article is supported by the project Deus ex Machina: NORTE - 01 - 0145 - FEDER - 000026 , supported by Norte Portugal Regional Operational Programme (NORTE 2020), under the PORTUGAL 2020 Partnership Agreement, through the European Regional Development Fund (ERDF) and by national funds through FCT - Fundação para a Ciência e Tecnologia within the Project Scope: UID/CEC/00319/2019. Vinicius Silva also thanks FCT for the $\mathrm{PhD}$ scholarship SFRH/BD/SFRH/BD/133314/2017.

\section{REFERENCES}

Moore, R., Lopes, J., 1999. Paper templates. In TEMPLATE'06, 1st International Conference on Template Production. SCITEPRESS. 
Smith, J., 1998. The book, The publishing company. London, $2^{\text {nd }}$ edition.

BISFed. (2017). BISFed International Boccia Rules (v.2).

Burchell, A. (2006). The Importance of Sport to the Disabled. The Commonwealth Health Minister's Book.

Calado, A., Leite, P., Soares, F., Novais, P., \& Arezes, P. (2018). Real-Time Gesture Classification for Monitoring Elderly Physical Activity Using a Wireless Wearable Device. The Ninth International Conference on Sensor Device Technologies and Applications, 164-168.

Calado, A., Leite, P., Soares, F., Novais, P., \& Arezes, P. (2019). Design of a Framework to Promote Physical Activity for the Elderly. In T. Ahram, W. Karwowski, \& R. Taiar (Eds.), Human Systems Engineering and Design (pp. 589-594). Cham: Springer International Publishing.

Calado, A., Marcutti, S., Vercelli, G., \& Novais, P. (2019). Developing an Intelligent Virtual Coach for Boccia : Design of a Virtual Boccia Simulator. 15th AAATE Conference Global Challenges in Assistive Technology Conference Global Challenges in Assistive Technology [In Press].

Calado, A., Silva, V., Soares, F., \& Novais, P. (2019). Ball Detection for Boccia Game Analysis. 2019 6th International Conference on Control, Decision and Information Technologies (CoDIT), 1468-1473.

CRE Discapacidad y Dependencia. (2016). Boccia Virtual. Retrieved February 5, 2019, from http://www.crediscapacidadydependencia.es/cresan andres_01/documentacion/bocciavirtual/index.htm

Donnelly, P., \& Kidd, B. (2006). Literature Reviews on Sport for Development. Sport for Development \& Peace. International Working Group, 1-195.

Edwards, P. (2012). Boccia. Retrieved February 5, 2019, from https://preloaded.com/work/boccia/

Eyck, A., Geerlings, K., Karimova, D., Meerbeek, B., Wang, L., IJsselsteinjn, W., ... Weterink, J. (2006). Effect of a Virtual Coach on Athletes' Motivation. International Conference on Persuasive Technology 2006, 158-161.

Figueira, C., Silva, J., Santos, A., Sousa, F., Silva, V., Ramos, J., ... Arezes, P. (2017). iBoccia: Monitoring elderly while playing Boccia gameplay. ICINCO 2017 - Proceedings of the 14th International Conference on Informatics in Control, Automation and Robotics, 1(Icinco), 670-675. https://doi.org/10.5220/0006473606700675

Fina, V. Della, Cera, R., \& Palmisano, G. (2017). The United Nations convention on the rights of persons with disabilities: A commentary. In The United Nations Convention on the Rights of Persons with Disabilities: A Commentary. https://doi.org/10.1007/978-3-319-43790-3

Guedelha, H. (2007). Boccia, o simulador. 96.

Kelly, P., Healy, A., Moran, K., \& O'Connor, N. E. (2010). A virtual coaching environment for improving golf swing technique. Proceedings of the 2010 ACM Workshop on Surreal Media and Virtual Cloning -
$S M V C$

'10,

51.

https://doi.org/10.1145/1878083.1878098

Leite, P., Calado, A., \& Soares, F. (2018). Boccia Court Analisys for Real-time Scoring. Proceedings of the 15th International Conference on Informatics in Control, Automation and Robotics, 2, 511-516. https://doi.org/10.5220/0006918305110516

Lowe, S., \& ÓLaighin, G. (2012). The age of the virtual trainer. 9th Conference of the International Sports Engineering Association (ISEA), 34, 242-247. https://doi.org/10.1016/j.proeng.2012.04.042

Machado Ribeiro, J. D. (2017). Boccia Game Simulator Applications for Training Cerebral Palsy Patients. Faculty of Engineering of the University of Porto. Retrieved from https://paginas.fe.up.pt/ ee11264/Tese/assets/JoseD iogoRibeiro_Dissertacao_MIEEC_VersaoProvisori a.pdf

Microsoft. (2017). Developing with Kinect.

Mixamo. (2019). Mixamo.

Osório, B., \& Sá, P. (2008). BOCCIASIM. Retrieved February 5, 2019, from http://bocciasim.blogspot.com

Pierson, J. (2009). Tackling social exclusion. Routledge.

Ruttkay, Z., \& Van Welbergen, H. (2008). Elbows higher! Performing, observing and correcting exercises by a virtual trainer. Lecture Notes in Computer Science (Including Subseries Lecture Notes in Artificial Intelligence and Lecture Notes in Bioinformatics), 5208 LNAI, 409-416. https://doi.org/10.1007/978-3540-85483-8 41

Silva, V., Ramos, J., Soares, F., Novais, P., Arezes, P., Sousa, F., ... Santos, A. (2018). iBoccia: A Framework to Monitor the Boccia Gameplay in Elderly. Lecture Notes in Computational Vision and Biomechanics, 27. https://doi.org/10.1007/978-3319-68195-5

UN. (2013). World Economic and Social Survey 2013: Sustainable Development Challenges. In United Nations, Department for Economic and Social Affairs.

https://doi.org/10.1016/j.urolonc.2009.06.002

United Nations. (2006). Convention on the rights of persons with disabilities. https://doi.org/10.1057/palgrave.development.1100 310

Unity. (2019). Unity Real-Time Development Platform 3D, 2D VR \& AR Visualizations.

Verweirder, W. (2015). wouterverweirder/kinect2: Nodejs library to access the kinect 2 data from the official MS SDK.

Watson, A., Bickmore, T., Cange, A., Kulshreshtha, A., \& Kvedar, J. (2012). An internet-based virtual coach to promote physical activity adherence in overweight adults: Randomized controlled trial. Journal of Medical Internet Research, 14(1). https://doi.org/10.2196/jmir.1629

World Health Organization. (2011). WORLD REPORT ON DISABILITY 